\title{
Nonlinear Intersubband Photoabsorption in Asymmetric Single Quantum Wells
}

\author{
H. O. Wijewardane* and C. A. Ullrich* \\ ${ }^{*}$ Department of Physics, University of Missouri-Rolla, Rolla, Missouri 65409, USA
}

\begin{abstract}
A density-matrix approach combined with time-dependent density-functional theory is used to calculate the intersubband photoabsorption in a strongly driven, DC-biased GaAs/AlGaAs single quantum well. For certain frequencies and intensities of the driving field, optical bistability is observed. Compared to a full time propagation of the density matrix, the commonly used two-level rotating wave approximation becomes less and less accurate for increasing asymmetry.
\end{abstract}

Intersubband (ISB) transitions in semiconductor quantum wells take place on a meV energy scale and are therefore attractive for $\mathrm{THz}$ device applications [1]. Nonlinear ISB dynamics has attracted particular attention, and many interesting effects have been studied: secondand third-harmonic generation [2], intensity-dependent saturation of photoabsorption [3, 4], directional control over photocurrents [5], generation of ultrashort $\mathrm{THz}$ pulses [6], plasma instability [7], or optical bistability $[8,9]$. Inspired by the photoabsorption experiments by Craig et al. [4], we have recently performed a theoretical study of the optical bistability region in a strongly driven, modulation $n$-doped $\mathrm{GaAs} / \mathrm{Al}_{0.3} \mathrm{Ga}_{0.7}$ As quantum well [10]. We have demonstrated that ISB bistability can be manipulated on a picosecond time scale by short $\mathrm{THz}$ control pulses. This opens up new opportunities for experimental study of optical bistability, which in the long run may lead to new $\mathrm{THz}$ applications such as high-speed all-optical modulators and switches.

Most previous theoretical studies of nonlinear ISB dynamics were based on the semiconductor Bloch equations (SBE) in Hartree [11]-[13] or exchange-only [14][16] approximation. These studies showed that the collective ISB electron dynamics is strongly influenced by depolarization and exchange-correlation (xc) manybody effects. We account for these effects using timedependent density-functional theory, which has the advantage of formal and computational simplicity.

The present study deals with a popular simplification of the ISB SBE: the 2-level rotating-wave approximation (RWA) [11]-[13], which was used by Załużny [11] to derive analytical expressions for nonlinear ISB photoabsorption. The 2-level RWA works well for symmetric quantum wells, but we will demonstrate numerically that it breaks down when the system becomes asymmetric under the influence of DC electric fields.
The conduction subbands are described in effectivemass approximation for GaAs, where $m^{*}=0.067 m$ and $e^{*}=e / \sqrt{\varepsilon}, \varepsilon=13$, are the effective mass and charge. The ground state is characterized by single-particle states $\Psi_{j \mathbf{q}_{\| \mid}^{0}}^{0}(\mathbf{r})=A^{-1 / 2} e^{i \mathbf{q}_{||} \mathbf{r}_{\|}} \psi_{j}^{0}(z)$, with $\mathbf{r}_{\|}$and $\mathbf{q}_{\|}$in the $x-y$ plane. The envelope function for the $j$ th subband $\psi_{j}^{0}(z)$ follows self-consistently from a one-dimensional KohnSham equation [17], with the ground-state density

$$
n(z)=2 \sum_{j, \mathbf{q}_{\|}}\left|\psi_{j}^{0}(z)\right|^{2} \theta\left(\varepsilon_{F}-E_{j q_{\|}}\right) .
$$

Here, $E_{j q_{\|}}=\varepsilon_{j}+\hbar^{2} q_{\|}^{2} / 2 m^{*}$, and $\varepsilon_{j}$ and $\varepsilon_{F}$ are the subband and Fermi energy levels. We consider electronic sheet densities $N_{s}$ such that only the lowest subband is occupied, in which case $\varepsilon_{F}=\pi \hbar^{2} N_{s} / m^{*}+\varepsilon_{1}$.

Under the influence of $\mathrm{THz}$ driving fields, linearly polarized along $z$, the time-dependent states have the form $\Psi_{j \mathbf{q}_{\| \mid}}(\mathbf{r}, t)=A^{-1 / 2} e^{i \mathbf{q}_{\| \mid} \mathbf{r}_{\| \mid}} \psi_{j}(z, t)$, with initial condition $\Psi_{j \mathbf{q}_{\| \mid}}\left(\mathbf{r}, t_{0}\right)=\Psi_{j \mathbf{q}_{\| \mid}^{0}}^{0}(\mathbf{r})$. The time-dependent Hamiltonian $H(t)=-\frac{\hbar^{2}}{2 m^{*}} \frac{\partial^{2}}{\partial z^{2}}+v_{\mathrm{qw}}(z)+v_{\mathrm{dr}}(z, t)+v_{\mathrm{H}}(z, t)+v_{\mathrm{xc}}(z, t)$

features $v_{\mathrm{dr}}(z, t)=e F z f(t) \sin (\omega t)$ describing the driving field, with electric field amplitude $F$, frequency $\omega$, and envelope $f(t) . v_{\mathrm{qw}}(z)$ is the bare quantum well potential, the Hartree potential $v_{\mathrm{H}}$ follows from Poisson's equation, and we use the time-dependent local-density approximation for $v_{\mathrm{xc}}[10]$. The time-dependent density $n(z, t)$ follows by substituting $\psi_{j}(z, t)$ in Eq. (1).

To account for disorder or phonon scattering, we use a density-matrix approach. We expand the first conduction subband as $\psi_{1}(z, t)=\sum_{k=1}^{N_{b}} c_{k}(t) \psi_{k}^{0}(z)$. The associated $N_{b} \times N_{b}$ density matrix $\rho$ has elements $\rho_{k l}(t)=c_{k}^{*}(t) c_{l}(t)$ 

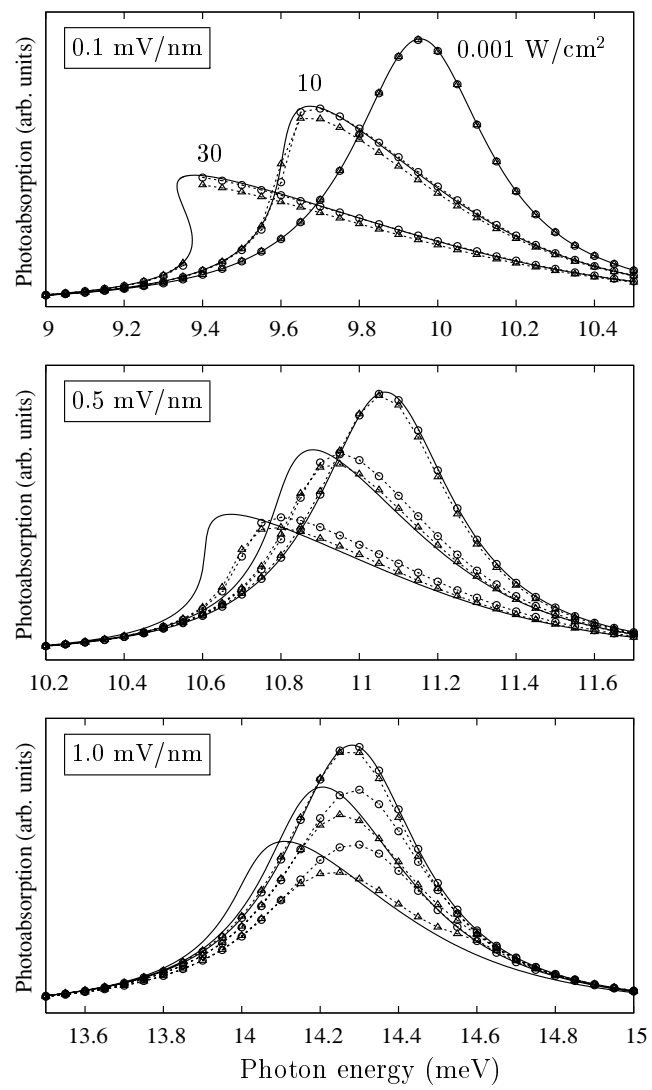

FIGURE 1. ISB photoabsorption for a $40 \mathrm{~nm} \mathrm{GaAs} / \mathrm{AlGaAs}$ quantum well with electron density $6.4 \times 10^{10} \mathrm{~cm}^{-2}$ and DC bias $0.1,0.5$, and $1.0 \mathrm{mV} / \mathrm{nm}$, driven by $\mathrm{THz}$ fields with intensities as indicated. Lines: 2-level RWA [11]. Symbols: full 2-level (circles) and 6-level (triangles) density-matrix propagation.

and initial condition $\rho_{k l}\left(t_{0}\right)=\delta_{k l} \delta_{1 k}$. The time evolution of $\rho$ follows from

$$
i \hbar \frac{\partial \rho(t)}{\partial t}=[H(t), \rho(t)]-R
$$

with the relaxation matrix $R_{k l}=\hbar\left[\rho_{k l}(t)-\rho_{k l}\left(t_{0}\right)\right] / T_{k l}$. For simplicity, $T_{k l}=T_{1} \delta_{k l}+T_{2}\left(1-\delta_{k l}\right)$, where $T_{1}$ and $T_{2}$ are phenomenological relaxation and decoherence times.

We consider a $40 \mathrm{~nm} \mathrm{GaAs} / \mathrm{Al}_{0.3} \mathrm{Ga}_{0.7}$ As square quantum well with $N_{s}=6.4 \times 10^{10} \mathrm{~cm}^{-2}$ [4], with $\varepsilon_{2}-\varepsilon_{1}=$ $8.72 \mathrm{meV}$ and ISB plasmon frequency $9.91 \mathrm{meV}$ at zero bias. We use $T_{1}=40 \mathrm{ps}$ and $T_{2}=3.1 \mathrm{ps}$, consistent with recent values for similar systems [17]-[19]. In the following, we apply DC electric fields $0.1,0.5$, and 1.0 $\mathrm{mV} / \mathrm{nm}$, and we perform 2-level and 6-level densitymatrix calculations $\left(N_{b}=2,6\right)$. To describe ISB photoabsorption, we propagate Eq. (3) in the presence of $\mathrm{THz}$ driving fields, switched on at $t_{0}$ over a 5-cycle linear ramp and then kept at constant intensity for several hundred ps.
The photoabsorption cross section (the dissipated power) $\sigma(\omega)$ follows from the induced dipole moment [10].

Figure 1 shows results for the ISB photoabsorption, comparing Załużny's 2-level RWA [11] with our density matrix calculations, for $\mathrm{THz}$ intensities $0.001,10$ and $30 \mathrm{~W} / \mathrm{cm}^{2}$. At low intensities, $\sigma(\omega)$ has a Lorentzian shape, and the RWA and full calculations agree very well. The ISB plasmon peak Stark-shifts to higher frequencies under DC bias $[17,18]$. At higher intensities, deviations from the Lorentzian lineshape are observed: population transfer into higher levels reduces the depolarization shift, predominantly at the peak position. At $0.1 \mathrm{mV} / \mathrm{nm}$ and $30 \mathrm{~W} / \mathrm{cm}^{2}$ this leads to bistability [10].

The 2-level RWA [11] and the full density-matrix calculations are close for small asymmetries, but discrepancies develop at increasing DC bias: the RWA tends to exaggerate the shift and change of shape of the absorption peak. The reason is that the RWA ignores all higher harmonics of $\omega$ in the induced density matrix, and thus the coupling to the diagonal matrix elements of the time-dependent potential, which are finite for asymmetric wells. We also observe more pronounced deviations between the 2-level and 6-level density-matrix calculations at larger asymmetries. A more complete analysis of the breakdown of the RWA for asymmetric systems will be presented in a forthcoming publication.

The authors acknowledge support from the donors of the Petroleum Research Fund, administered by the ACS. C.A.U. is a Cottrell Scholar of the Research Corporation.

\section{REFERENCES}

1. Intersubband Transitions in Quantum Wells I, edited by $\mathrm{H}$. C. Liu and F. Capasso, Semiconductors and Semimetals Vol. 62 (Academic Press, San Diego, 2000).

2. J. N. Heyman et al., Phys. Rev. Lett. 72, 2183 (1994).

3. F. H. Julien et al., Appl. Phys. Lett. 53, 116 (1988).

4. K. Craig et al., Phys. Rev. Lett. 76, 2382 (1996).

5. E. Dupont et al., Phys. Rev. Lett. 74, 3596 (1995).

6. J. N. Heyman et al., Appl. Phys. Lett. 72, 644 (1998).

7. P. Bakshi et al., Appl. Phys. Lett. 75, 1685 (1999).

8. M. Seto and M. Helm, Appl. Phys. Lett. 60, 859 (1992).

9. M. I. Stockman et al., Phys. Rev. B 48, 10966 (1993).

10. H. O. Wijewardane and C. A. Ullrich, Appl. Phys. Lett. 84, 3984 (2004).

11. M. Załużny, Phys. Rev. B 47, 3995 (1993); J. Appl. Phys. 74, 4716 (1993).

12. B. Galdrikian and B. Birnir, Phys. Rev. Lett. 76, 3308 (1996).

13. A. A. Batista et al., Phys. Rev. B 66, 195325 (2002).

14. D. E. Nikonov et al., Phys. Rev. Lett. 79, 4633 (1997).

15. A. Olaya-Castro et al., Phys. Rev. B 68, 155305 (2003).

16. J. Li and C. Z. Ning, Phys. Rev. Lett. 91, 097401 (2003).

17. C. A. Ullrich and G. Vignale, Phys. Rev. B 58, 15756 (1998); Phys. Rev. Lett. 87, 037402 (2001).

18. J. B. Williams et al., Phys. Rev. Lett. 87, 037401 (2001).

19. J. N. Heyman et al., Phys. Rev. Lett. 74, 2682 (1995). 